\title{
Relating the quark and gluon condensates through the QCD vacuum energy
}

\author{
E. V. Gorbar* and A. A. Natale ${ }^{\dagger}$ \\ Instituto de Física Teórica, Universidade Estadual Paulista, Rua Pamplona, 145, 01405, São Paulo, SP, Brazil
}

(Received 7 June 1999; revised manuscript received 2 September 1999; published 9 February 2000)

\begin{abstract}
Using the Cornwall-Jackiw-Tomboulis effective potential for composite operators we compute the QCD vacuum energy as a function of the dynamical quark and gluon propagators, which are related to their respective condensates as predicted by the operator product expansion. The identification of this result to the vacuum energy obtained from the trace of the energy-momentum tensor allows us to study the gluon self-energy, verifying that it is fairly represented in the ultraviolet by the asymptotic behavior predicted by the operator product expansion, and in the infrared it is frozen at its asymptotic value at one scale of the order of the dynamical gluon mass. We also discuss the implications of this identity for heavy and light quarks. For heavy quarks we recover, through the vacuum energy calculation, the relation $m_{f}\left\langle\bar{\psi}_{f} \psi_{f}\right\rangle \sim-\frac{1}{12}\left(\alpha_{s} / \pi\right) G^{\mu \nu} G_{\mu \nu}$ obtained many years ago with QCD sum rules.
\end{abstract}

PACS number(s): 12.38.-t, 11.30.Qc, 11.30.Rd

\section{INTRODUCTION}

The QCD vacuum energy can be computed through the use of the effective potential for composite operators [1]. This vacuum energy is a function of the masses of the theory $[1,3]$. For quarks the effective mass is a sum of the running mass and the dynamical one and the quark condensate is an order parameter for chiral symmetry breaking (see, e.g., Ref. [4]). According to the operator product expansion (OPE) $[5,6]$ the dynamical mass is a function of the quark condensate. Therefore, the vacuum energy involves the bare quark masses and the quark condensate.

The concept of a dynamical gluon mass [7] is crucial to calculate the QCD vacuum energy. (We would like to emphasize that the presence of a dynamically generated mass does not mean that gluons can be considered as massive asymptotic states similar to dynamically generated quark mass does not mean that quarks can be observed as massive asymptotic states. Why quarks and gluons are not observed as free states is the well known problem of confinement.) As discussed at length in Ref. [7], the vacuum energy is finite as long as the gluon mass decreases at large momentum, and it vanishes when the gluon mass is zero. The infrared divergences that plagued the effective potential calculations are also absent for a finite dynamical gluon mass. Finally, the gluon mass is related to the gluon condensate, and a precise relation, as in the case of the dynamical quark mass, has been obtained through the OPE [8]. It is also known that the gluon contribution dominates the vacuum energy. However, not much is known about the infrared behavior of the dynamical gluon mass.

On the other hand the vacuum energy can also be obtained through the trace of the energy momentum tensor and is a function of the quark and gluon condensates and the bare

\footnotetext{
* On leave of absence from Bogolyubov Institute for Theoretical Physics, Metrologichna 14-b, 252143, Kiev, Ukraine. Email address: gorbar@ift.unesp.br

${ }^{\dagger}$ Email address: natale@ift.unesp.br
}

quark mass. Equalizing these two expressions we obtain information about the dynamical masses of the theory and the condensates. In this work we use two different calculations of the vacuum energy density in order to determine the behavior of the dynamical masses, particularly of the gluon dynamical mass. We show that it is fairly represented in the ultraviolet by the asymptotic behavior predicted by the OPE, and in the infrared it is equal to its value at a scale of the order of the dynamical gluon mass. Within this approach we are also able to obtain a relation between the quark and gluon condensates in the heavy quark limit. It is opportune to recall that many years ago Shifman et al. introduced the notion of a nonvanishing gluon condensate [9]. Making use of the OPE they started the successful program of QCD sum rules, where observable quantities can be written as a function of quark and gluon condensates, and one of their main results is the relation between quark and gluon condensates valid in the heavy quark limit [10]:

$$
m_{f}\left\langle\bar{\psi}_{f} \psi_{f}\right\rangle \sim-\frac{1}{12}\left\langle\frac{\alpha_{s}}{\pi} G^{\mu \nu} G_{\mu \nu}\right\rangle .
$$

This relation emerges naturally in our calculation of the vacuum energy. We also discuss what can be learned from our approach in the chiral and light fermion limits. In Sec. II we setup the equations for the vacuum energy. Section III contains a discussion of our computational Ansätze for the dynamical masses, and Sec. IV contains the vacuum energy calculation. In the last section we discuss the method and present the conclusions.

\section{THE EFFECTIVE POTENTIAL FOR COMPOSITE OPERATORS AND THE QCD VACUUM ENERGY}

For a non-Abelian gauge theory the effective potential has the form [1] 

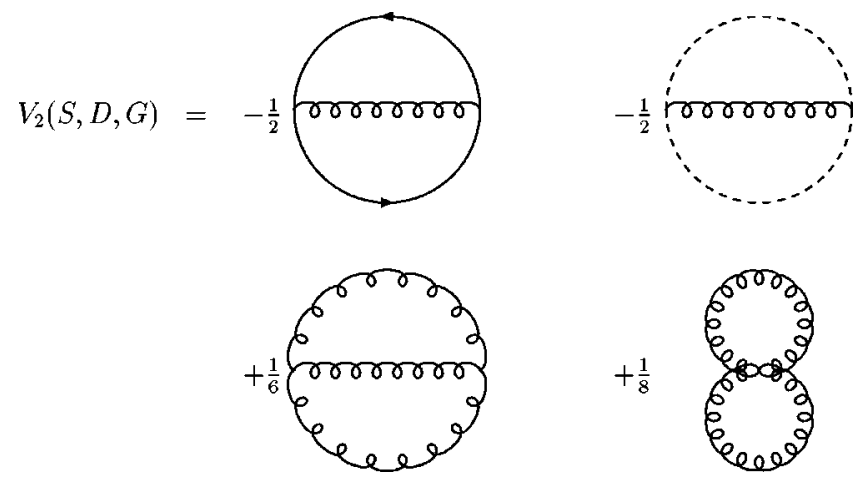

FIG. 1. Two-particle irreducible vacuum diagrams contributing to the effective potential.

$$
\begin{aligned}
V(S, D, G)= & -\imath \int \frac{d^{4} p}{(2 \pi)^{4}} \operatorname{Tr}\left(\ln S_{0}^{-1} S-S_{0}^{-1} S+1\right) \\
& -\imath \int \frac{d^{4} p}{(2 \pi)^{4}} \operatorname{Tr}\left(\ln G_{0}^{-1} G-G_{0}^{-1} G+1\right) \\
& +\frac{\imath}{2} \int \frac{d^{4} p}{(2 \pi)^{4}} \operatorname{Tr}\left(\ln D_{0}^{-1} D-D_{0}^{-1} D+1\right) \\
& +V_{2}(S, D, G)
\end{aligned}
$$

where $S, D$, and $G$ are the complete propagators of fermions, gauge bosons, and Faddeev-Popov ghosts, respectively; $S_{0}$, $D_{0}$, and $G_{0}$ the corresponding bare propagators. $V_{2}(S, D, G)$ is the sum of all two-particle irreducible vacuum diagrams, depicted in Fig. 1, and the equations

$$
\frac{\delta V}{\delta S}=\frac{\delta V}{\delta D}=\frac{\delta V}{\delta G}=0
$$

are the Schwinger-Dyson equations (SDE) for fermions, gauge bosons, and ghosts.

We can represent $V_{2}(S, D, G)$ analytically by

$$
\begin{aligned}
\iota V_{2}(S, D, G)= & -\frac{1}{2} \operatorname{Tr}(\Gamma S \Gamma S D)-\frac{1}{2} \operatorname{Tr}(F G F G D) \\
& +\frac{1}{6} \operatorname{Tr}\left(\Gamma^{(3)} D \Gamma^{(3)} D D\right)+\frac{1}{8} \operatorname{Tr}\left(\Gamma^{(4)} D D\right),
\end{aligned}
$$

where $\Gamma, F, \Gamma^{(3)}$, and $\Gamma^{(4)}$ are, respectively, the proper vertices of fermions, ghosts, trilinear and quartic gauge boson couplings [2]. In Eq. (4) we have not written the gauge and Lorentz indices, as well as the momentum integrals.

The complete gauge boson propagator $D$ is related to the free propagator by

$$
D^{-1}=D_{0}^{-1}-\Pi^{T}
$$

where $\Pi^{T}$ is the gluon polarization tensor, which is obtained from Eqs. (2) and (3), and described by

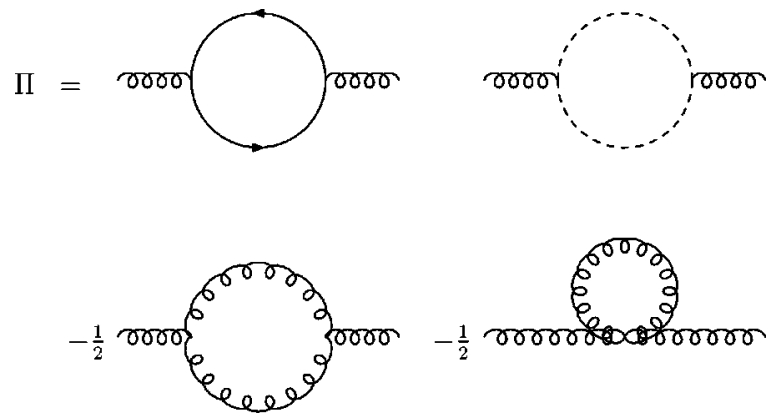

FIG. 2. Diagrams contributing to the gluon polarization tensor.

$$
\Pi^{T}=\Gamma S \Gamma S+F G F G-\frac{1}{2} \Gamma^{(3)} D \Gamma^{(3)} D-\frac{1}{2} \Gamma^{(4)} D .
$$

The diagrams contributing to $\Pi^{T}$ are shown in Fig. 2, and this self-energy is the one that admits a massive solution as described by Cornwall [7]. The Landau gauge expression for the complete gluon propagator is

$$
D^{\mu \nu}\left(p^{2}\right)=-\frac{l}{p^{2}-\Pi\left(p^{2}\right)}\left(g^{\mu \nu}-\frac{p^{\mu} p^{\nu}}{p^{2}}\right) .
$$

The complete fermion propagator $S$ is related to the free propagator by

$$
S^{-1}=S_{0}^{-1}-\Sigma
$$

where $S_{0}={ }_{\imath} /\left(\not p-m_{0}\right)\left(m_{0}\right.$ is the bare quark mass) and $\Sigma$ is the Schwinger-Dyson equation for the quark self-energy, which is given by

$$
\Sigma=-\gamma S \Gamma D
$$

where $\gamma$ is the bare vertex.

The vacuum energy density is given by the effective potential calculated at minimum subtracted by its perturbative part, which does not contribute to dynamical mass generation $[1,3,7]$

$$
\langle\Omega\rangle=V_{\min }(S, D, G)-V_{\min }\left(S_{p}, D_{p}, G_{p}\right),
$$

where $S_{p}$ is the perturbative counterpart of $S$, etc.

$V_{\min }$ is obtained substituting the solutions of Eqs. (3) into (2). It is opportune to recall that we shall compute the vacuum energy by using Ansätze for the dynamical masses. Although these Ansätze are consistent with the OPE they are simple approximations to the full solution of the SchwingerDyson equations. However, since we calculate the potential at its stationary point, the result depends significantly less on the approximations used, as was noticed for the vacuum energy calculation in the case of fermions [11]. If we use the corresponding Schwinger-Dyson equations, $V_{\min }$ can be divided into two parts (as will become clear in the following)

$$
V_{\min }=V_{\min }^{f}+V_{\min }^{g},
$$

where the labels $f$ and $g$ indicate the fermionic and gluonic parts of the vacuum energy. The quark contribution to the 
effective potential obtained by substituting the solution of $\delta V / \delta S=0$ into Eq. (2) and (in Euclidean space with $P^{2}$ $\left.\equiv-p^{2}\right)$ is equal to [11]

$$
V_{\min }^{f}=2 N \int \frac{d^{4} P}{(2 \pi)^{4}}\left[-\ln \left(\frac{P^{2}+\Sigma^{2}}{P^{2}+m_{0}^{2}}\right)+\frac{\Sigma^{2}-\Sigma m_{0}}{P^{2}+\Sigma^{2}}\right],
$$

where $N$ is the number of colors [quarks are in the fundamental representation of $\mathrm{SU}(N)]$.

To compute $V_{\min }^{g}$ we make an approximation which considerably simplifies the calculation. This approximation frequently performed to solve the gluon polarization tensor is due to Mandelstam [12] and consists in neglecting the ghosts and the diagram with quartic coupling. The neglect of ghosts diagrams was shown to be reasonable, because their contribution is numerically small [12]. These approximations, with the use of the Landau gauge, were shown to be satisfactory in the lengthy and detailed work of Brown and Pennington [13]. Although it may seem rough this approximation has been verified to be phenomenologically consistent in Ref. [14] as long as the vacuum polarization tensor involves a mass scale. Furthermore, as can be easily verified, by expanding the integrand in Eq. (15) in powers of $\Pi$ we obtain a result similar to the Cornwall determination of the vacuum energy in the case of a pure gluon theory [7]. Therefore, only the diagram with trilinear coupling should be considered in Eq. (4), i.e., the gluon polarization tensor is given by

$$
\Pi^{T}=-\frac{1}{2} \Gamma^{(3)} D \Gamma^{(3)} D
$$

With this approximation the two-loop gluon contribution in Eq. (4) is reduced to

$$
V_{2}^{g}=\frac{-\imath}{6} \operatorname{Tr}\left(\Gamma^{(3)} D \Gamma^{(3)} D D\right)
$$

Finally, we obtain [14]

$$
\begin{aligned}
\left\langle\Omega_{g}\right\rangle= & -\frac{3\left(N^{2}-1\right)}{2} \int \frac{d^{4} P}{(2 \pi)^{4}}\left[\frac{\Pi}{P^{2}+\Pi}-\ln \left(1+\frac{\Pi}{P^{2}}\right)\right. \\
& \left.+\frac{2}{3} \frac{\Pi^{2}}{P^{2}\left(P^{2}+\Pi\right)}\right],
\end{aligned}
$$

where all the quantities are in Euclidean space and $N=3$ for QCD.

Thus, according to Eq. (10), the vacuum energy is

$$
\langle\Omega\rangle=\left\langle\Omega_{f}\right\rangle+\left\langle\Omega_{g}\right\rangle,
$$

where $\left\langle\Omega_{f}\right\rangle$ is [see Eqs. (10) and (12)]

$$
\begin{aligned}
\left\langle\Omega_{f}\right\rangle= & 2 N \int \frac{d^{4} P}{(2 \pi)^{4}}\left[-\ln \left(\frac{P^{2}+\Sigma^{2}}{P^{2}+m_{0}^{2}}\right)+\frac{\Sigma^{2}-\Sigma m_{0}}{P^{2}+\Sigma^{2}}\right] \\
& -2 N \int \frac{d^{4} P}{(2 \pi)^{4}}\left[-\ln \left(\frac{P^{2}+m_{f}^{2}}{P^{2}+m_{0}^{2}}\right)+\frac{m_{f}^{2}-m_{f} m_{0}}{P^{2}+m_{f}^{2}}\right],
\end{aligned}
$$

where $m_{f}^{2} \equiv m_{f}^{2}\left(P^{2}\right)$ is the running quark mass, which is the perturbative solution of the fermionic Schwinger-Dyson equation with bare quark mass $m_{0}$. Note that Eq. (17) has been written for a unique fermion of mass $m_{f}$. If we consider $n_{f}$ fermions of the same mass it should be obviously multiplied by $n_{f}$. There is only one divergence in $\left\langle\Omega_{f}\right\rangle$ that is associated to the bare quark mass renormalization as will be discussed afterwards. For gluons with dynamically generated mass the gluon contribution to the vacuum energy is free of divergences $[7,14]$. As long as we have appropriate expressions for $\Sigma$ and $\Pi$ the calculation of $\langle\Omega\rangle$ is an easy task.

\section{THE SELF-ENERGY OF QUARKS AND GLUONS}

To compute the vacuum energy we need reasonable $A n$ sätze for the quark and gluon self-energies. In the case of quarks the high energy behavior of the dynamical mass given by the OPE is $[5,6]$

$$
m_{\mathrm{dyn}}\left(P^{2}\right) \simeq \frac{3\left(N^{2}-1\right)}{8 N^{2}} g^{2}\left(P^{2}\right) \frac{\langle-\bar{\psi} \psi\rangle}{P^{2}}\left(\ln P^{2} / \Lambda_{Q}^{2}\right)^{-\gamma_{m}},
$$

where $g^{2}\left(P^{2}\right)$ is the running coupling constant

$$
g^{2}\left(P^{2}\right)=\frac{2 b}{\ln P^{2} / \Lambda_{Q}^{2}},
$$

where $b=24 \pi^{2} /\left(11 N-2 n_{f}\right)$. In the framework of SDE the same behavior was found in Ref. [15]. As well known the asymptotic behavior of the running quark mass is the following:

$$
m_{f}^{\text {asymp }}\left(P^{2}\right) \sim m_{f}\left(\mu^{2}\right)\left(\frac{\ln P^{2} / \Lambda_{Q}^{2}}{\ln \mu^{2} / \Lambda_{Q}^{2}}\right)^{-\gamma_{m}},
$$

where $\Lambda_{Q}$ is the QCD scale, $\gamma_{m}=9 C_{2} /\left(11 N-2 n_{f}\right), C_{2}$ $=\left(N^{2}-1\right) / 2 N$ is the quadratic Casimir of the fundamental representation, and $m_{f}\left(\mu^{2}\right)$ is the running quark mass at the scale $\mu^{2}$ (a mass scale where perturbative QCD can be safely applied), and is related to the bare mass by

$$
m_{f}(\mu)=m_{0}(\Lambda) Z_{m}^{-1}(\mu, \Lambda),
$$

where $\Lambda$ is a ultraviolet cutoff and $Z_{m}^{-1}(\mu, \Lambda)$ is a renormalization constant for the bare mass term

$$
Z_{m}^{-1}(\mu, \Lambda)=\left(\frac{\ln \mu^{2} / \Lambda_{Q}^{2}}{\ln \Lambda^{2} / \Lambda_{Q}^{2}}\right)^{\gamma_{m}} .
$$


A discussion of the renormalization procedure applied to the effective potential for composite operators can be found in Ref. [16].

Equations (18) and (20) involve the high momentum part of the self-energy. To establish our Ansatz for $\Sigma$ for all $P^{2}$, we recall a few points on the infrared behavior of the quark mass. There are several arguments in favor of the relation [17]

$$
\Sigma\left(P^{2} \rightarrow 0\right) \approx\langle-\bar{\psi} \psi\rangle^{1 / 3}
$$

for massless or very light quarks. For heavy quarks we have

$$
\left.\Sigma\left(P^{2} \rightarrow 0\right) \approx m_{f}\left(P^{2}\right)\right|_{P^{2}=m_{f}^{2}}
$$

These equations provide an idea about the Ansatz we should use for the quark self-energy. Actually, there is a full numerical solution of the quark Schwinger-Dyson equations with bare massive quarks performed by Maris and Roberts [18], and they verify that for very heavy quarks the self-energy is given by Eq. (24) at low momentum and behaves as Eq. (20) for a momentum scale larger than the current mass, i.e., the contribution of dynamically generated mass to the total mass is negligible for heavy quarks. For light quarks $\left(m_{f}\right.$ $<\langle-\bar{\psi} \psi\rangle^{1 / 3}$ ) the self-energy is a sum of the dynamical mass which dominates in the low momentum region but the asymptotic behavior of self-energy is still given by Eq. (20). Only at the chiral limit $\Sigma$ is fully described by the dynamical mass function. This behavior does not change when we consider gluons with a dynamically generated mass [19], although we obtain smaller values for the dynamical quark masses (at $P^{2} \rightarrow 0$ ) in this case [20,21]. For large gluon masses the signal of chiral symmetry breaking may disappear when we solve the Schwinger-Dyson equation in the lowest order [21], but we consider this as a failure of the method for solving gap equations [20] at the lowest order and still assume that Eqs. (18) and (23) are valid.

Our Ansatz based on the solutions of Ref. [18] has the form

$$
\Sigma\left(P^{2}\right)=m_{f}\left(P^{2}\right)+m_{\mathrm{dyn}}\left(P^{2}\right),
$$

which is a sum of the bare and dynamical mass. According to the numerical solutions of Ref. [18] and in agreement with the Ansatz already used in Refs. [11,16] we can have the following expressions.

(a) In the chiral limit (CL)

$$
\Sigma_{\mathrm{CL}}\left(P^{2}\right)=m_{\mathrm{dyn}}\left(P^{2}\right),
$$

where

$$
\begin{aligned}
m_{\mathrm{dyn}}\left(P^{2}\right)= & \eta^{3}\left[\frac{1}{\eta^{2}} \theta\left(\eta^{2}-P^{2}\right)+\frac{1}{P^{2}}\left(\frac{\ln P^{2} / \Lambda_{Q}^{2}}{\ln \eta^{2} / \Lambda_{Q}^{2}}\right)^{\gamma_{m}-1}\right. \\
& \left.\times \theta\left(P^{2}-\eta^{2}\right)\right],
\end{aligned}
$$

where $\eta$ is a mass scale which in principle can be related to the quark condensate, since in QCD, according to Eq. (18), at some large mass scale $\left(\mu \gg \Lambda_{Q}\right)$ we must have $\eta^{3}$ $\propto\left[3\left(N^{2}-1\right) g^{2}\left(\mu^{2}\right) / 8 N^{2}\right]\langle-\bar{\psi} \psi\rangle_{\mu}$. The above Ansatz is equal to the one used in Ref. [16], where the full effective potential was computed and $\eta$ was considered a variational parameter to be phenomenologicaly adjusted. In our case we calculate the minimum of energy. Therefore, our Ansatz must approach as close as possible the actual solution of the Schwinger-Dyson equation. Consequently, to be also compatible with the low energy phenomenology [Eq. (23)], which sets a scale for all the chiral observables, we set

$$
\eta \equiv\langle-\bar{\psi} \psi\rangle_{\eta}{ }^{1 / 3} .
$$

(b) In the case of a light fermion ( $l f$ ) (i.e., $m_{f}<\eta$ ) we define

$$
\begin{aligned}
m_{l f}\left(P^{2}\right)= & m_{f}\left(\eta^{2}\right)\left[\theta\left(\eta^{2}-P^{2}\right)+\left(\frac{\ln P^{2} / \Lambda_{Q}^{2}}{\ln \eta^{2} / \Lambda_{Q}^{2}}\right)^{-\gamma_{m}}\right. \\
& \left.\times \theta\left(P^{2}-\eta^{2}\right)\right],
\end{aligned}
$$

which has the correct low and high energy behavior, and use the Ansatz

$$
\Sigma_{l f}=m_{l f}\left(P^{2}\right)+m_{\mathrm{dyn}}\left(P^{2}\right) .
$$

The numerical solutions of the Schwinger-Dyson equations [18] show that when the bare mass is much smaller than the dynamical mass, $\Sigma\left(P^{2}\right)$ starts falling as $1 / P^{2}$ just after $P^{2}$ $\approx \eta^{2}$, changing to the logarithmic behavior when $\eta^{3} / P^{2}$ becomes of the order of the bare mass. Equation (30) reproduces this behavior and can reasonably represent the masses of the quarks $u, d$, and $s$ with a suitable choice of bare masses.

(c) For heavy fermions $(h f)$ we introduce

$$
m_{h f}\left(P^{2}\right)=m_{f}\left[\theta\left(m_{f}^{2}-P^{2}\right)+\left(\frac{\ln P^{2} / \Lambda_{Q}^{2}}{\ln m_{f}^{2} / \Lambda_{Q}^{2}}\right)^{-\gamma_{m}} \theta\left(P^{2}-m_{f}^{2}\right)\right],
$$

where the basic difference with Eq. (29) is the choice of arguments of the $\theta$ functions. From the results of Ref. [18] we could be tempted to set $\Sigma_{h f}\left(P^{2}\right)=m_{h f}$, which is of course the leading contribution to $\Sigma\left(P^{2}\right)$ for very heavy quarks. However, notice that Eq. (17) would give $\left\langle\Omega_{f}\right\rangle=0$ with this approximation, and we must stick to Eq. (25) to obtain the correct result as will be discussed in the next section.

Equations (25)-(31) give a quite reasonable fit for the full numerical solutions of the quark Schwinger-Dyson equations. The $\theta$ functions separate the regions where techniques such as the OPE and renormalization group are reliable from the ones where nonperturbative effects are present and we can at most rely on phenomenological models. Being a variational parameter the scale $\eta$ can be adjusted in order to fit observable chiral parameters [16]. Moreover, the freezing of 
the coupling constant at the scale $\eta$ is also implicit in Eq. (27). Although there are arguments in the literature showing that this freezing may already occur at the gluon mass scale $[20,22]$, we do not expect substantial differences in our calculation due to this approximation as will be discussed in the conclusion.

We can now turn to the Ansatz for the gluon polarization tensor. The behavior of $\Pi$ away from the high momentum region is much less known and is more controversial. Nonperturbative solutions for the gluon propagator have been searched for a long time, but only recently it became more common to talk about a possible infrared finite behavior of this propagator. As discussed in Ref. [14] there are several forms proposed in the literature for the gluon polarization tensor in the infrared, which appear due to different approximations performed when solving the Schwinger-Dyson equation for the gluon propagator, and there is an indication that the vacuum energy is minimized for gluons with a dynamically generated mass [14]. A strong support for this possibility comes from the gluon propagator simulation in lattice QCD [23]. These results may be considered preliminary and large lattice studies must be pursued, but they definitively show signals of a gluon mass scale up to reasonably low momenta [23]. We shall assume here that gluons have indeed a dynamical mass as predicted by Cornwall [7] many years ago, and to obtain an Ansatz for the gluon polarization tensor we are guided by the OPE again. The OPE can teach us only about the high energy behavior of the dynamical mass which is given by [8]

$$
\Pi_{\mathrm{OPE}}\left(P^{2}\right) \sim-\frac{34 N \pi^{2}}{9\left(N^{2}-1\right)} \frac{\left\langle\left(\alpha_{s} / \pi\right) G^{\mu \nu} G_{\mu \nu}\right\rangle}{P^{2}} .
$$

For the infrared we assume that $\Pi$ freezes at the value predicted by Eq. (32) at some scale which should be determined. This freezing is consistent with the prediction of Ref. [7] of a constant gluon mass in the infrared. Therefore, we assume

$$
\Pi\left(P^{2}\right)=\mu_{g}^{2} \theta\left(\chi \mu_{g}^{2}-P^{2}\right)+\frac{\mu_{g}^{4}}{P^{2}} \theta\left(P^{2}-\chi \mu_{g}^{2}\right),
$$

where

$$
\mu_{g}^{2}=\left(\frac{34 N \pi^{2}}{9\left(N^{2}-1\right)}\left\langle\frac{\alpha_{s}}{\pi} G^{\mu \nu} G_{\mu \nu}\right\rangle\right)^{1 / 2} .
$$

Notice that $\chi$ in Eq. (33) is a variational parameter to be determined in our calculation. It defines the scale that separates the perturbative and nonperturbative regions [24]. This choice of Ansatz is fully based on the result of the operator product expansion for the gluon propagator [8] and on the phenomenological estimates of the dynamical gluon mass $[7,14]$. Note that this is an Ansatz in the case of a pure gluon theory. Of course, fermions modify this result. There are two types of fermionic contributions to the dynamical gluon mass. One is proportional to the gluon condensate but appears only at the next order in the coupling constant, i.e., the gluon mass is going to be modified by a factor (1 $\left.+\alpha_{s} n_{f} \cdots\right)$. Therefore, for heavy quarks their contribution is small due to the smallness of the coupling constant at the scale of heavy quarks. However, for light fermions this is no longer true. Unfortunately there is not enough information on the infrared behavior of the gluon propagator to do better than this. Therefore, we are forced to consider phenomenologically the contribution of fermions. The other contribution to the gluon mass is proportional to the fermion condensate and its mass $m_{f}\langle\bar{\psi} \psi\rangle$, which could be important for heavy quarks but as we checked is numerically small compared to the contribution proportional to the gluon condensate and pure gluon loop. Equations (25) and (33) are the basic ingredients of the vacuum energy computation that we present in the next section.

\section{THE GLUON SELF-ENERGY AND A RELATION BETWEEN THE QUARK AND GLUON CONDENSATES}

As discussed in the beginning our main goals are to study the infrared behavior of the gluon propagator and obtain a relation between the quark and gluon condensates similar to Eq. (1) through the vacuum energy analysis. For this we recall that the vacuum expectation value of the trace of the energy momentum tensor of QCD is [25]

$$
\left\langle\Theta_{\mu \mu}\right\rangle=\frac{\beta(g)}{2 g}\left\langle G_{\mu \nu} G^{\mu \nu}\right\rangle-\sum_{f} m_{f}\langle-\bar{\psi} \psi\rangle\left(1+\gamma_{m}\right),
$$

where the perturbative $\beta(g)$ function up to two loops is

$$
\begin{aligned}
\frac{\beta(g)}{2 g}= & -\frac{1}{24} \frac{\alpha_{s}}{\pi}\left[\left(11 N-2 n_{f}\right)+\frac{1}{4} \frac{\alpha_{s}}{\pi}\left(34 N^{2}-10 N n_{f}\right.\right. \\
& \left.\left.-3 n_{f} \frac{N^{2}-1}{N}\right)\right]
\end{aligned}
$$

with $\alpha_{s}=g^{2}(\mu) / 4 \pi$. Equation (35) is related to the vacuum energy through

$$
\left\langle\Omega^{(\mathrm{tr})}\right\rangle=\frac{1}{4}\left\langle\Theta_{\mu \mu}\right\rangle .
$$

When we compare this last expression for $\langle\Omega\rangle$ with the one of Sec. II we can fix the behavior of the dynamical gluon mass as well as we can obtain a relation between the quark and gluon condensates.

The calculation of the vacuum energy using the ansatze of the previous section is straightforward. However, it is more convenient to present the results of the calculation in different parts. The gluon contribution to the vacuum energy is obtained substituting Eq. (33) into Eq. (15), and the result is

$$
\begin{aligned}
\left\langle\Omega_{g}\right\rangle= & -\frac{3\left(N^{2}-1\right)}{32 \pi^{2}}\left[\frac{\chi-1}{2}-\frac{1}{3} \ln (\chi+1)-\left(\frac{1}{6}+\chi^{2}\right) \ln \chi\right. \\
& \left.+\left(\frac{3 \chi^{2}+2}{6}\right) \ln \left(\chi^{2}+1\right)\right] \mu_{g}^{4},
\end{aligned}
$$


where $\mu_{g}$ is given by Eq. (34).

Because of the form of the Ansatz for the quark selfenergy its contribution to the vacuum energy has to be computed only numerically. In the chiral limit we set $m_{0}=m_{f}$ $=0$ into Eq. (17) and easily verify that

$$
\begin{aligned}
\left\langle\Omega_{f}\right\rangle_{\mathrm{CL}}= & -\frac{N \eta^{4}}{16 \pi^{2}}\left[2 \ln 2-1+2 \int_{1}^{\Lambda^{2} / \eta^{2}} d z z(\ln (1+h / z)\right. \\
& \left.\left.-\frac{h / z}{1+h / z}\right)\right]
\end{aligned}
$$

where $h$ is given by

$$
h=z^{-2}\left(\frac{\ln \left(z \eta^{2} / \Lambda_{Q}^{2}\right)}{\ln \left(\eta^{2} / \Lambda_{Q}^{2}\right)}\right)^{2 \gamma_{m}-2} .
$$

When we consider bare massive fermions there is an essential modification in the calculation due to the mass renormalization. We initially consider the case where $m_{f} \ll \eta$ and work with the Ansatz of Eq.(30). Expanding the high energy part in powers of $1 / P^{2}$, we verify that Eq. (17) reduces to

$$
\begin{aligned}
\left\langle\Omega_{f}\right\rangle_{l f} \simeq & -\frac{N}{8 \pi^{2}}\left[\int_{0}^{\eta^{2}} d z z\left(\ln \frac{z+\left(\eta+m_{f}\right)^{2}}{z+m_{f}^{2}}-\frac{\eta\left(\eta+m_{f}\right)}{z+\eta^{2}}\right)\right. \\
& +m_{0}(\Lambda) \eta^{3} \int_{\eta^{2}} \frac{\Lambda^{2}}{z}\left(\ln \frac{z}{\Lambda_{Q}^{2}}\right)^{\gamma_{m}-1} \\
& \left.+\int_{\eta^{2}}^{\Lambda^{2}} d z z\left(\ln \left(1+h \eta^{2} / z\right)-\frac{h \eta^{2} / z}{1+h \eta^{2} / z}\right)\right] \\
\simeq & -\frac{N}{8 \pi^{2}}\left[\int_{0}^{\eta^{2}} d z z\left(\ln \frac{z+\left(\eta+m_{f}\right)^{2}}{z+m_{f}^{2}}-\frac{\eta\left(\eta+m_{f}\right)}{z+\eta^{2}}\right)\right. \\
& +\frac{\langle-\bar{\psi} \psi\rangle_{\eta^{m}} m_{f}(\eta)}{\gamma_{m}}+\int_{\eta^{2}}^{\Lambda^{2}} d z z\left(\ln \left(1+h \eta^{2} / z\right)\right. \\
& \left.\left.-\frac{h \eta^{2} / z}{1+h \eta^{2} / z}\right)\right],
\end{aligned}
$$

where we kept only the leading term in $m_{f}$ in the ultraviolet part as well as the terms independent of the bare mass. A numerical evaluation of the above expression shows that $\left\langle\Omega_{f}\right\rangle_{l f}$ has a very small variation with $m_{f}$ up to masses of the order of $100 \mathrm{MeV}$ when we assume $\eta \sim 230 \mathrm{MeV}$ with the minimum becoming $5 \%$ deeper for masses of this order.

For very heavy fermions $\left(m_{f} \gg \eta\right)$ the Schwinger-Dyson equations tell us that $[18,19] \Sigma\left(P^{2}\right) \approx m_{f}\left(P^{2}\right)$, and it is not difficult to see that the fermion mass effect is basically erased from the vacuum energy Eq. (17) apart from a term coming from the mass renormalization, which survives no matter how small is the contribution from the dynamical mass. Therefore, for each heavy fermion of mass $m_{f}$ we obtain

$$
\left\langle\Omega_{f}\right\rangle_{h f} \simeq-\frac{N\langle-\bar{\psi} \psi\rangle_{\eta} m_{f}(\eta)}{8 \pi^{2} \gamma_{m}} .
$$

Note that the renormalization group invariant quantity $m_{f}\langle-\bar{\psi} \psi\rangle$ appears after the mass renormalization. For light fermions the terms proportional to the bare mass in $\left\langle\Omega_{f}\right\rangle_{l f}$ are significantly smaller in comparison with the terms due to the dynamical mass. For heavy fermions, i.e., for masses larger than a few $\mathrm{GeV}$, all terms in $\left\langle\Omega_{f}\right\rangle_{h f}$ other than $m_{f}$ $\langle-\bar{\psi} \psi\rangle$ can be neglected without affecting considerably our forthcoming numerical estimates.

To determine the parameter $\chi$ which separates the perturbative and nonperturbative regions of the gluon self-energy and to find a relation between the condensates, we consider the equality

$$
\left\langle\Omega^{(t r)}\right\rangle=\left\langle\Omega_{g}\right\rangle+\left\langle\Omega_{f}\right\rangle .
$$

We fix the parameter $\chi$ by working with the theory without fermions. We obtain the following expression:

$$
\begin{aligned}
\frac{\beta(g)}{8 g}\left\langle G_{\mu \nu} G^{\mu \nu}\right\rangle= & -\frac{3\left(N^{2}-1\right)}{32 \pi^{2}} \mu_{g}^{4}\left(\frac{\chi-1}{2}-\frac{1}{3} \ln (\chi+1)\right. \\
& -\left(\frac{1}{6}-\chi^{2}\right) \ln \chi+\left(\frac{3 \chi^{2}+2}{6}\right) \\
& \left.\times \ln \left(\chi^{2}+1\right)\right)
\end{aligned}
$$

Substituting $m_{g}$ given by Eq. (34) and considering the $\beta(g)$ function up to one loop we obtain

$$
\begin{gathered}
{\left[\frac{\chi-1}{2}-\frac{1}{3} \ln (\chi+1)-\left(\frac{1}{6}+\chi^{2}\right) \ln \chi\right.} \\
\left.+\left(\frac{3 \chi^{2}+2}{6}\right) \ln \left(\chi^{2}+1\right)\right]=\frac{11}{34},
\end{gathered}
$$

which gives

$$
\chi \approx 0.966797
$$

This value, being so close to 1 , means that the gluon mass given by Eq. (34) is a reasonable scale to separate the perturbative and nonperturbative behaviors of the gluon propagator. In the following we assume a complete cancellation of $\left\langle\Omega_{g}\right\rangle$ with the part of $\left\langle\Omega^{(\operatorname{tr})}\right\rangle$ coming from the gluonic contribution to the $\beta(g)$ function when we use the above value of $\chi$. The phenomenological value of the gluon mass obtained in this way is also consistent with previous estimates [7]. It is important to recall that in the OPE calculation of the gluon polarization tensor fermions do not contribute at leading order to the term proportional to $\left\langle\left(\alpha_{s} / \pi\right) G^{\mu \nu} G_{\mu \nu}\right\rangle$, and this cancelation is indeed complete.

We can now consider the presence of fermions. The case of heavy quarks is the simplest one. Using Eqs. (37), (38), and (42) and taking into account the cancelation mentioned above, for $n_{f}$ heavy fermions of equal mass we obtain 


$$
\begin{aligned}
& \frac{n_{f}}{48}\left\langle\frac{\alpha_{s}}{\pi} G^{\mu \nu} G_{\mu \nu}\right\rangle-\frac{n_{f} m_{f}}{4}\langle-\bar{\psi} \psi\rangle\left(1+\gamma_{m}\right) \\
& \quad=-\frac{N n_{f}\langle-\bar{\psi} \psi\rangle_{\eta} m_{f}(\eta)}{8 \pi^{2} \gamma_{m}}
\end{aligned}
$$

entailing

$$
m_{f}\langle-\bar{\psi} \psi\rangle \approx \frac{1}{\kappa}\left\langle\frac{\alpha_{s}}{\pi} G^{\mu \nu} G_{\mu \nu}\right\rangle .
$$

Neglecting the second term of the $\beta$ function, what is totally acceptable because $\alpha_{s}$ is small on the scale of heavy quarks, we obtain

$$
\kappa=12\left(1+\gamma_{m}-\frac{N}{2 \pi^{2} \gamma_{m}}\right),
$$

which gives $\kappa \approx 11.9,12.6,13.2$, respectively, for one, two, and three heavy quark flavors. Thus, by calculating the vacuum energy as a function of the quark and gluon condensates we have reproduced the old result of Ref. [10]. Note that this result appears naturally due to the cancellation between the gluonic parts of the vacuum energy and the fact that we can neglect the heavy quark contribution to the gluonic part of the effective potential, because the heavy quark contribution to the gluon mass proportional to the gluon condensate appears in the next order of $\alpha_{s}$. We would like to note that the nonleading terms neglected in the operator product expansion, for example, the gluon condensate contribution to the quark self-energy $\Sigma \propto m_{f}\left\langle\left(\alpha_{s}\right)\right.$ $\left.\pi) G^{\mu \nu} G_{\mu \nu}\right\rangle / P^{4}$, and vice versa the quark condensate contribution to the gluon polarization $\Pi \propto m_{f}\langle-\bar{\psi} \psi\rangle / P^{2}$ may slightly modify our results, but even if we were intended to introduce them in the calculation we should remember that we do not have a reliable phenomenology to correctly describe their behavior at low momenta.

The identity between the two calculations of the vacuum energy can also provide some information on the gluon propagator in the case of massless fermions. The physically interesting case is the one where the bare mass is zero but the chiral symmetry is broken dynamically. It is known that chiral symmetry breaking happens when the gauge coupling exceeds a critical value

$$
\alpha_{c}=\frac{\pi}{3 C_{2}} .
$$

In principle this value (or a larger one [27,28,21]) should be introduced in the $\beta(g)$ in Eq. (52). Unfortunately we do not know the behavior of the $\beta$ function in this region. In this case we will use Eq. (36) at the extreme of its domain of validity, which is the best that we can do at the moment. However, we still have another problem because our Ansatz for the gluon polarization is valid only at leading order in $\alpha_{s}$ (we do not explicitly take into account the fermionic contribution to the gluon polarization tensor, while we need to compare it to the two-loop $\beta$ function). We proceed in a different way to the case of heavy fermions. For heavy fermions we used the identity between two ways of calculating the vacuum energy to obtain a relation between the condensates. Here, vice versa, we use it to obtain information on the Ansatz for the gluon polarization. In what follows we modify our Ansatz for the gluon mass performing the following replacement in Eq. (34):

$$
\mu_{g}^{4} \rightarrow \mu_{g}^{4}\left(1+\alpha_{s} n_{f} c\right)
$$

where $c$ is another parameter to be determined through Eq. (43). The parametrization in Eq. (51) was chosen because the fermion contribution to the gluon mass proportional to the gluon condensate should depend on $n_{f}$ and $\alpha_{s}$.

In the chiral limit we have to compute numerically the integral in Eq. (39). Assuming that $\eta$ and $\Lambda_{Q}$ may vary around the values 0.2 and $0.3 \mathrm{GeV}$, we verified that the integral in this equation contributes at most $10 \%$ of the total value between the brackets of Eq. (39), i.e., only the low energy region contributes effectively to the vacuum energy. If we neglect the high energy part of $\left\langle\Omega_{f}\right\rangle$ and take into account the cancellation already discussed for the gluonic contributions in Eq. (43) we obtain for the chiral broken phase

$$
\begin{gathered}
-\frac{1}{96}\left\langle\frac{\alpha_{s}}{\pi} G^{\mu \nu} G_{\mu \nu}\right)\left[-2 n_{f}+\frac{\alpha_{s}}{4 \pi}\left(34 N^{2}-10 N n_{f}\right.\right. \\
\left.\left.-3 n_{f} \frac{N^{2}-1}{N}\right)\right] \\
\approx-\frac{3\left(N^{2}-1\right)}{32 \pi^{2}}\left[\frac{\chi-1}{2}-\frac{1}{3} \ln (\chi+1)\right. \\
\left.-\left(\frac{1}{6}-\chi^{2}\right) \ln \chi+\left(\frac{3 \chi^{2}+2}{6}\right) \ln \left(\chi^{2}+1\right)\right] \\
\quad \times \mu_{g}^{4}\left(1+\alpha_{s} n_{f} c\right)-\frac{N \eta^{4}(2 \ln 2-1)}{16 \pi^{2}} .
\end{gathered}
$$

With $n_{f}=1, \quad \alpha_{s} \sim \alpha_{c}, \quad \chi \quad$ given by Eq. (46), $\left\langle\left(\alpha_{s} / \pi\right) G^{\mu \nu} G_{\mu \nu}\right\rangle \simeq(0.01) \mathrm{GeV}^{4} \quad[10]$, and $\langle-\bar{\psi} \psi\rangle$ $\simeq(0.23 \mathrm{GeV})^{3}[29]$, we obtain $c \approx 8.7$. This large value of $c$ means that the next order term in Eq. (34) in the case of light fermions cannot be neglected. It would be interesting to compute the next order contribution to the dynamical gluon mass through the OPE including the effect of fermions and see how it compares to our estimate. Another numerical relation between the quark and gluon condensates could also be obtained for the case where the quarks have a small bare mass, using the values of $\chi$ and $c$ determined above and Eq. (41) for the fermionic contribution to the effective potential. However, such relation will be useful only with a better knowledge of the nonperturbative $\beta$ function and of the nextto-leading order determination of the gluon mass through the OPE. 


\section{CONCLUSIONS}

Starting from the effective action for composite operators [1] we determined the QCD vacuum energy as a function of the dynamical quark and gluon masses. The ultraviolet behavior of these masses can be related to the quark and gluon condensates through the operator product expansion, and the same is expected for their infrared parts, although, concerning this low energy behavior, we do have to rely on phenomenological data. With this procedure we obtain the vacuum energy described as a function of the condensates and bare quark masses. Equalizing this expression to the one obtained from the trace of the energy momentum tensor we were able to fix the behavior of the dynamical gluon mass. We verified that the gluon polarization tensor is fairly well represented by the Ansatz we have chosen [Eq. (33)] and that the gluon mass given by Eq. (34) is a reasonable scale to separate perturbative and nonperturbative behaviors of the gluon propagator. It is remarkable that our simple ansatz for the dynamical gluon mass gives such a good result.

For heavy fermions we reproduced the old result of QCD sum rules $m_{f}\langle-\bar{\psi} \psi\rangle \approx(1 / 11.9)\left\langle\left(\alpha_{s} / \pi\right) G^{\mu \nu} G_{\mu \nu}\right\rangle \quad[10]$ which in our case comes naturally from the effective potential calculation. Note that this formula has been derived at one-loop and is essentially similar to the result of Shifman et al. in the sense that it is a consequence of the behavior of two-point functions. However, the concept of dynamical gluon mass is relevant here, without it we cannot compute an effective potential free of ambiguities (as a spurious infrared cutoff). We also discussed how the ansatz for the dynamical gluon mass could be improved and how it can be tested with the next-to-leading order calculation of the OPE.

The method proposed here can be improved in several ways. We certainly need a description of the $\beta$ function in the nonperturbative region, without this we do not believe that we can go far away with these calculations in the light fermion domain. The other improvements are related to the approximations that we have performed to compute $\langle\Omega\rangle$, i.e., the choice of the Ansätze for quark and gluon propagators. The computation of $\left\langle\Omega_{g}\right\rangle$ has shown to be quite reliable [14], and in spite of neglecting the contribution of diagrams with quadrilinear vertices and ghosts, the final result is similar to the Hartree approximation made by Cornwall in his calculation of the vacuum energy [7], and both calculations reduce the two-loop contributions to a term whose main contribution to the effective potential is proportional to $\Pi^{2}$. In a more rigorous calculation the two-loop effective potential should be calculated including all diagrams, as well as the full vertices because they are fundamental for cancellations present in the full calculation [26]. As we computed the effective potential at the minimum of energy we escaped from this very difficult task, and also minimized the effect of the approximations [11].

Another point that could be discussed at length is the question of the dynamical masses Ansätze. It is obvious that the desirable scenario would be a full numerical solution of the Schwinger-Dyson equations concomitantly with the effective potential, but this is also an enormous work. The Ansätze we used are in agreement with the phenomenology at low momenta and the OPE at high momenta. Notice that the important point is the connection between the dynamical masses and the condensates, and it is not relevant what mechanism generates the condensates or masses as long as the relation predicted by the OPE between the condensates and the masses holds.

Finally, the idea of the calculation is very simple, and we were able to recover, within the approximations of this method, the relation between quark and gluon condensates for heavy quarks obtained by Shifman et al. many years ago [10], and verify that the vacuum energy is well described by a dynamically massive gluon, whose momentum dependence is displayed in Eqs. (33) and (34).

\section{ACKNOWLEDGMENTS}

We would like to thank V.P. Gusynin for valuable remarks. This research was supported by the Conselho Nacional de Desenvolvimento Científico e Tecnológico (CNPq) (A.A.N.), by Fundacão de Amparo à Pesquisa do Estado de São Paulo (FAPESP) (E.G.,A.A.N.), and by Programa de Apoio a Núcleos de Excelência (PRONEX).
[1] J. M. Cornwall, R. Jackiw, and E. Tomboulis, Phys. Rev. D 10, 2428 (1974).

[2] J. M. Cornwall and R. C. Shellard, Phys. Rev. D 18, 1216 (1978); A. A. Natale, Nucl. Phys. B226, 365 (1983).

[3] J. M. Cornwall and R. E. Norton, Phys. Rev. D 8, 3338 (1973).

[4] P. I. Fomin, V. P. Gusynin, V. A. Miransky, and Yu. A. Sitenko, Riv. Nuovo Cimento 6, 1 (1983).

[5] H. D. Politzer, Nucl. Phys. B117, 397 (1976).

[6] P. Pascual and E. de Rafael, Z. Phys. C 12, 127 (1982).

[7] J. M. Cornwall, Phys. Rev. D 26, 1453 (1982).

[8] M. Lavelle, Phys. Rev. D 44, R26 (1991).

[9] M. A. Shifman, A. I. Vainshtein, M. B. Voloshin, and V. I. Zakharov, Phys. Lett. 77B, 80 (1978); A. Vainshtein, V. Zakharov, and M. Shifman, Pis'ma Zh. Eksp. Teor. Fiz. 27, 60 (1978) [JETP Lett. 27, 55 (1978)].
[10] M. A. Shifman, A. I. Vainshtein, and V. I. Zakharov, Nucl. Phys. B147, 385 (1979); B147, 448 (1979).

[11] P. Castorina and S.-Y. Pi, Phys. Rev. D 31, 411 (1985); J. C. Montero, A. A. Natale, V. Pleitez, and S. F. Novaes, Phys. Rev. D 161, 151 (1985); V. P. Gusynin and Yu. A. Sitenko, Z. Phys. C 29, 547 (1985).

[12] S. Mandelstam, Phys. Rev. D 20, 3223 (1979).

[13] N. Brown and M. R. Pennington, Phys. Rev. D 38, 2266 (1988); 39, 2723 (1989).

[14] J. C. Montero, A. A. Natale, and P. S. Rodrigues da Silva, Phys. Lett. B 406, 130 (1997).

[15] V. A. Miransky, Sov. J. Nucl. Phys. 38, 280 (1983); Phys. Lett. 165B, 401 (1985); K. Higashijima, Phys. Rev. D 29, 1228 (1984).

[16] A. Barducci et al., Phys. Rev. D 38, 238 (1988). 
[17] B. H. J. McKellar, M. D. Scadron, and R. C. Warner, Int. J. Mod. Phys. A 3, 203 (1988).

[18] P. Maris and C. D. Roberts, preprint Argonne Lab. ANL-PHY8842-97, Proceedings of the 4th International Workshop on Progress in Heavy Quark Physics, Rostock 1997, pp. 159-162, nucl-th/9710062; see also C. D. Roberts, Fiz. B 8, 285 (1999).

[19] P. S. Rodrigues da Silva, Ph.D. thesis, IFT/UNESP, 1998.

[20] J. Papavassiliou and J. M. Cornwall, Phys. Rev. D 44, 1285 (1991).

[21] J. C. Montero, A. A. Natale, and P. S. Rodrigues da Silva, Prog. Theor. Phys. 96, 1209 (1996); A. A. Natale and P. S. Rodrigues, Phys. Lett. B 390, 378 (1997); 392, 444 (1997).

[22] J. M. Cornwall and J. Papavassiliou, Phys. Rev. D 40, 3474 (1989).

[23] UKQCD Collaboration, D. B. Leinweber et al., Phys. Rev. D 60, 094507 (1999); 58, 031501 (1998); C. Bernard, C. Parrinello, and A. Soni, ibid. 49, 1585 (1994); P. Marenzoni, G. Martinelli, N. Stella, and M. Testa, Phys. Lett. B 318, 511
(1993); P. Marenzoni et al., Report No. QCD162:Q83:1994, 1994, pp. 210-212; R. Gupta et al., Phys. Rev. D 36, 2813 (1987); J. E. Mandula and M. Ogilvie, Phys. Lett. B 185, 127 (1987); P. A. Amundsen and J. Greensite, ibid. 173, 179 (1986); C. Bernard, ibid. 108, 431 (1982).

[24] A. A. Natale and P. S. Rodrigues da Silva, Phys. Lett. B 442, 369 (1998).

[25] R. Crewther, Phys. Rev. Lett. 28, 1421 (1972); M. Chanowitz and J. Ellis, Phys. Lett. 40B, 397 (1972); J. C. Collins, A. Duncan, and S. D. Joglekar, Phys. Rev. D 16, 438 (1977).

[26] J. M. Cornwall, Phys. Rev. D 10, 500 (1974).

[27] D. Atkinson and P. W. Johnson, Phys. Rev. D 37, 2296 (1988); C. D. Roberts and B. H. J. McKellar, ibid. 41, 672 (1990).

[28] C. D. Roberts and A. G. Williams, in Progress in Particle and Nuclear Physics, edited by A. Faessler (Pergamon, Oxford, 1994), Vol. 33, p. 477.

[29] H. G. Dosch and S. Narison, Phys. Lett. B 417, 173 (1998). 